\title{
Higher twist corrections to Bjorken sum rule
}

\author{
Mauro Anselmino \\ Dipartimento di Fisica Teorica, Università di Torino \\ and Istituto Nazionale di Fisica Nucleare, Sezione di Torino \\ Via P. Giuria 1, I-10125 Torino, Italy \\ Francisco Caruso* and Eugene Levin** \\ Centro Brasileiro de Pesquisas Físicas/CNPq \\ Rua Dr. Xavier Sigaud 150, 22290-180, Rio de Janeiro, Brazil
}

\begin{abstract}
Some higher twist corrections to the Bjorken sum rule are estimated in the framework of a quark-diquark model of the nucleon. The parameters of the model have been previously fixed by fitting the measured higher twist corrections to the unpolarized structure function $F_{2}\left(x, Q^{2}\right)$. The resulting corrections to the Bjorken sum rule turn out to be negligible.
\end{abstract}

* Also at Physics Institute of the Universidade do Estado do Rio de Janeiro, 20550-013, Rio de Janeiro, Brazil

** On leave of absence from Theory Department of Petersburg Nuclear Physics Institute, 188350, St. Petersburg, Gatchina, Russia 
Several recent measurements of the polarized structure functions $g_{1}^{p}(x)$ and $g_{1}^{n}(x)$ [1] both for protons and neutrons have allowed the first tests of the fundamental Bjorken sum rule [2]:

$$
\int_{0}^{1} d x\left[g_{1}^{p}(x)-g_{1}^{n}(x)\right]=S_{B j}=\frac{a_{3}}{6} E_{N S}\left(Q^{2}\right)
$$

where $a_{3}=1.2573 \pm 0.0028$ is the neutron $\beta$-decay axial coupling. The above equation holds on ground of isospin invariance, at leading twist in the Operator Product Expansion; the coefficient function $E_{N S}$ has been computed up to third order [3] in the strong coupling constant $\alpha_{s}$ :

$$
E_{N S}\left(Q^{2}\right)=1-\frac{\alpha_{s}}{\pi}-3.58\left(\frac{\alpha_{s}}{\pi}\right)^{2}-20.22\left(\frac{\alpha_{s}}{\pi}\right)^{3}
$$

where a number of three active flavours is assumed.

Data are available at $Q^{2}=2$ and $4.6(\mathrm{GeV} / \mathrm{c})^{2}[1,4,5]$ for the L.H.S. of Eq. (1): $0.152 \pm 0.014 \pm 0.021$ and $0.201 \pm 0.039 \pm 0.050$, respectively. At such values of $Q^{2}$ a comparison of Bjorken sum rule with experiment should also take into account possible higher twist or target mass corrections, not included in Eq. (1):

$$
\int_{0}^{1} d x\left[g_{1}^{p}(x)-g_{1}^{n}(x)\right]=S_{B j}\left(Q^{2}\right)+\delta S_{H T}^{B j}+\delta S_{T}^{B j}
$$

Some evaluations of target mass [6-8] and higher twist corrections can be found in the literature [1], either in the framework of the Drell-Hearn-Gerasimov sum rule [9] or of the QCD sum rules $[6,10]$. Some of these corrections may be sizeable at $Q^{2} \simeq 2(\mathrm{GeV} / \mathrm{c})^{2}$, but the final comparison with data shows no significant violation of the Bjorken sum rule. Let us notice that reliable estimates of the higher twist contributions are of fundamental importance in view of the recent suggestions $[7,11]$ to exploit the Bjorken sum rule (1) in order to extract the value of the strong coupling constant $\alpha_{s}$.

To this purpose we consider here another possible evaluation of higher twist corrections to $S_{B j}$, in the framework of a quark-diquark model of the nucleon. Diquarks originate from QCD colour forces and model correlations between two quarks inside the nucleon which, at moderate $Q^{2}$ values, interact collectively and behave as bound states; diquarks effectively describe the attractive QCD forces 
between two quarks inside a baryon. Evidence in support of the diquark model is given by a number of phenomenological successes in the description of several physical phenomena, such as: the behaviour of the DIS structure functions at Bjorken $x \rightarrow 1$, the inclusive large $p_{T}$ deuteron production observed in $70 \mathrm{GeV} / \mathrm{c}$ $p p$ interaction at Serpukhov, the large $p_{T}$ baryon production in $p p$ interactions, the observed (but scarce) production of $\Delta^{++}$at large transverse momentum, and many other processes reviewed in Ref. [12]. It is also worthwhile mentioning that this approach has recently found some further theoretical support in the Random Instanton Liquid Model for the structure of QCD vacuum [13].

A most general version of the diquark model has been recently used to describe, with excellent results, the higher twist contributions to the unpolarized proton structure function $F_{2}\left(x, Q^{2}\right)$ [14]. Such fit has led to values of the parameters of the model in good agreement with several previous applications of simplified versions of the same model [12]. We would like to stress that the diquark approach, being a physically motivated self consistent model rather than an expansion in powers of $1 / Q^{2}$, has the unique feature of taking into account a full set of higher twist corrections and not only the leading ones. Confident in its physical content, we adopt here the same diquark model, with the same parameters, as in Ref. [14]; in that our results are now genuine, parameter free predictions.

We recall that when probing the nucleon, in a quark-diquark state $[14,15]$, with the virtual photon in DIS one has three kinds of contributions: the scattering off the single quarks outside the diquark, the elastic scattering off the diquark, and the inelastic diquark contribution, i.e., the scattering off one of the quarks inside the diquark. At large $Q^{2}$ values the diquark contributions, weighted by form factors, vanish and one recovers the usual pure quark results.

The elastic diquark contributions have been studied in Ref. [15] and, for the polarized structure function $g_{1}\left(x, Q^{2}\right)$ they read

$$
g_{1}^{(V)}\left(x, Q^{2}\right)=\frac{1}{4} e_{V}^{2} \Delta V(x)\left[\left(2+\frac{Q^{2}}{2 m_{N}^{2} x^{2}}\right)\left(D_{1} D_{2}+\frac{1}{2} Q^{2} D_{2} D_{3}\right)-\frac{Q^{2}}{4 m_{N}^{2} x^{2}} D_{2}^{2}\right]
$$

where $D_{1,2,3}$ are form factors appearing in the most general coupling of the photon to a spin 1 diquark; scalar diquarks obviously do not contribute to the polarized 
structure functions. $\Delta V$ denotes the difference between the number density of vector diquarks with spin parallel and antiparallel to the nucleon spin.

Following the notations of Ref. [14] the higher twist diquark contributions to $g_{1}$ for protons and neutrons are given by:

$$
\begin{aligned}
{\left[g_{1}^{H T}\right]_{p} } & =\frac{1}{4}\left(\frac{16}{9} \Delta V_{u u}+\frac{1}{9} \Delta V_{u d}\right)\left[\left(2+\frac{Q^{2}}{2 m_{N}^{2} x^{2}}\right)\left(D_{1} D_{2}+\frac{1}{2} Q^{2} D_{2} D_{3}\right)\right. \\
& \left.-\frac{Q^{2}}{4 m_{N}^{2} x^{2}} D_{2}^{2}\right]-\frac{1}{2}\left(\frac{4}{9} \Delta u_{V_{u u}}+\frac{4}{9} \Delta u_{V_{u d}}+\frac{1}{9} \Delta d_{V_{u d}}\right) D_{V}^{2} \\
{\left[g_{1}^{H T}\right]_{n} } & =\frac{1}{4}\left(\frac{1}{9} \Delta V_{u u}+\frac{1}{9} \Delta V_{u d}\right)\left[\left(2+\frac{Q^{2}}{2 m_{N}^{2} x^{2}}\right)\left(D_{1} D_{2}+\frac{1}{2} Q^{2} D_{2} D_{3}\right)\right. \\
& \left.-\frac{Q^{2}}{4 m_{N}^{2} x^{2}} D_{2}^{2}\right]-\frac{1}{2}\left(\frac{1}{9} \Delta u_{V_{u u}}+\frac{4}{9} \Delta u_{V_{u d}}+\frac{1}{9} \Delta d_{V_{u d}}\right) D_{V}^{2}
\end{aligned}
$$

where the suffices to $V$ indicate the quark content of the vector diquark; the negative contributions come from the scattering off the quarks inside the diquarks, weighted by a factor $\left(1-D_{V}^{2}\right)$, with $D_{V}$ related to the diquark form factor. In Eq. (6) we have already used isospin relationships and all distribution functions refer to the proton; $\Delta q$ is the usual helicity density carried by quark $q$ and the suffices refer to the type of diquark it comes from.

Subtracting Eqs. (6) from (5) yields

$$
\begin{aligned}
{\left[g_{1}^{H T}\right]_{p-n} } & =\frac{5}{12} \Delta V_{u u}\left[\left(2+\frac{Q^{2}}{2 m_{N}^{2} x^{2}}\right)\left(D_{1} D_{2}+\frac{1}{2} Q^{2} D_{2} D_{3}\right)-\frac{Q^{2}}{4 m_{N}^{2} x^{2}} D_{2}^{2}\right] \\
& -\frac{1}{6} \Delta u_{V_{u u}} D_{V}^{2} .
\end{aligned}
$$

In order to give numerical estimates we have to introduce explicit expressions for the polarized distribution functions and the diquark form factors; the valence quark content of the proton is assumed to be given by the flavour and spin wave function

$$
\begin{aligned}
\left|p, S_{z}= \pm 1 / 2\right\rangle & = \pm \frac{1}{3}\left\{\operatorname { s i n } \Omega \left[\sqrt{2} V_{(u d)}^{ \pm 1} u^{\mp}-2 V_{(u u)}^{ \pm 1} d^{\mp}\right.\right. \\
& \left.\left.+\sqrt{2} V_{(u u)}^{0} d^{ \pm}-V_{(u d)}^{0} u^{ \pm}\right] \mp 3 \cos \Omega S_{(u d)} u^{ \pm}\right\}
\end{aligned}
$$

where $\sin ^{2} \Omega$ and $\cos ^{2} \Omega$ are, respectively, the probabilities of finding a vector $(V)$ or a scalar $(S)$ diquark in the proton. According to the above equation we have

$$
\begin{aligned}
\Delta V_{u u}(x) & =\frac{4}{9} \sin ^{2} \Omega f_{V_{u u}}(x) \\
\Delta u_{V_{u u}}(x) & =\frac{8}{9} \sin ^{2} \Omega f_{u_{V_{u u}}}(x)
\end{aligned}
$$


where the $f$ distributions are normalized to $\int_{0}^{1} d x f(x)=1$.

From Eqs. (7), (9) and (3), upon integration over $x$ and taking, as in Ref. [14], $D_{1}=D_{2}=D_{V}$, we obtain

$$
\begin{aligned}
{\left[\delta S_{H T}^{B j}\right]_{\text {diquarks }} } & =\frac{\sin ^{2} \Omega}{27}\left\{6 D_{1}^{2}+5 Q^{2} D_{1} D_{3}\right. \\
& \left.+\frac{5 Q^{2}}{4 m_{N}^{2}}\left(D_{1}^{2}+Q^{2} D_{1} D_{3}\right) \int_{0}^{1} \frac{d x}{x^{2}} f_{V_{u u}}(x)\right\} .
\end{aligned}
$$

Eq. (10) gives the quark-diquark model higher twist contributions to Bjorken sum rule. We take the explicit expressions of the distribution function $f_{V_{u u}}$ for a vector diquark and of the form factors from our previous application of the same model to a fit of higher twist contributions to the unpolarized structure function $F_{2}\left(x, Q^{2}\right)[14]:$

$$
\begin{aligned}
\sin ^{2} \Omega & =0.19 \\
f_{V_{u u}} & =N x^{7.93}(1-x)^{3.32} \\
D_{1}=D_{2}=D_{V} & =\left(\frac{1.21}{1.21+Q^{2}}\right)^{2} \\
D_{3} & =\frac{Q^{2}}{m_{N}^{4}} D_{1}^{2}
\end{aligned}
$$

where $N=1 / B(7.93 ; 3.32)$ is the normalization constant ( $B$ is the Euler beta function).

From Eqs. (10) and (11) we obtain the numerical estimates at the $Q^{2}$ values [in $\left.(\mathrm{GeV} / \mathrm{c})^{2}\right]$ for which data are available:

$$
\begin{aligned}
& {\left[\delta S_{H T}^{B j}\left(Q^{2}=2.0\right)\right]_{\text {diquarks }} \simeq 0.3 \times 10^{-2}} \\
& {\left[\delta S_{H T}^{B j}\left(Q^{2}=4.6\right)\right]_{\text {diquarks }} \simeq 0.6 \times 10^{-3}}
\end{aligned}
$$

The prediction of the model in the full range $1 \leq Q^{2} \leq 10(\mathrm{GeV} / \mathrm{c})^{2}$ is shown in Fig 1.

It is interesting to compare the results given in Eqs. (12) not only with the experimental values of $S_{B j}$ [reported after Eq. (2)], but also with the perturbative QCD higher order corrections of Eqs. (1) and (2), namely with $a_{3}\left[E_{N S}\left(Q^{2}\right)-1\right] / 6$. This definitely shows that the higher twist contributions to the Bjorken sum rule, evaluated in the framework of the quark-diquark model of the nucleon, turn out to be quite small. Indeed our model yields a contribution which has an opposite 
sign with respect to the pQCD corrections, but much smaller in magnitude: at $Q^{2}=2$ and $4.6(\mathrm{GeV} / \mathrm{c})^{2}$ we have, respectively,

$$
\frac{\left[\delta S_{H T}^{B j}\left(Q^{2}\right)\right]_{\text {diquarks }}}{a_{3}\left[1-E_{N S}\left(Q^{2}\right)\right] / 6} \simeq 7 \% \text { and } 2 \%
$$

To better understand why it happens so, it might be instructive to compute the diquark contribution to the first moment of $g_{1}^{p}(x)$ alone, i.e., $\Gamma_{1}^{p}=\int_{0}^{1} d x g_{1}^{p}(x)$. A sizeable diquark contribution to $\Gamma_{1}^{p}$ would indicate that the tiny results obtained above for the Bjorken sum, Eqs. (12), are only due to a strong cancellation between the proton and the neutron contributions. However, an explicit calculation shows that diquark contributions to $\Gamma_{1}^{p}$ are comparable to those found for the Bjorken sum rule, rejecting this possibility. We can then safely conclude that the smallness of the diquark contributions to the polarized structure functions is due to some intrinsic features of the quark-diquark model of the nucleon: the strong $S U(6)$ violation ( $\sin ^{2} \Omega=0.19$ ) favouring scalar diquarks; the mass scale of the vector diquark form factor which turns out to be small, $Q_{V}^{2}=1.21(\mathrm{GeV} / \mathrm{c})^{2}$, corresponding to a large size, and the vector diquark $x$ distribution which is found to be peaked at $x \simeq 0.7$, suggesting that vector diquarks consist of two almost uncorrelated quarks. In conclusion we would like to repeat the main statement of our abstract, namely that diquark contributions to the Bjorken sum rules turns out to be quite small. We hope that our estimates will help in making the use of the Bjorken sum rule in the experimental measurement of the strong coupling constant more reliable.

Acknowledgements - We thank M. Karliner and J. Feltesse for many useful talks on the subject. One of us (M.A.) would like to thank the members of the LAFEX at $\mathrm{CBPF}$, where most of this work was done, for the kind hospitality. F.C. and E.L. are grateful to CNPq of Brazil for a financial support. 
Figure caption - The value of $\left[\delta S_{H T}^{B j}\right]_{\text {diquarks }}$, according to Eqs. (10) and (11) of the text, as a function of $Q^{2}$.

\section{References}

[1] For a complete list of references and a comprehensive review paper on the subject see, e.g., M. Anselmino, A. Efremov and E. Leader, CERN preprint CERN-TH/7216/94, to appear in Phys. Rep.

[2] J.D. Bjorken, Phys. Rev. 148 (1966) 1467

[3] S.A. Larin and J.A.M. Vermaseren, Phys. Lett. B259 (1991) 345; S.A. Larin, Phys. Lett. B334 (1994) 192

[4] B. Adeva et al., Phys. Lett. B302 (1993) 533; B320 (1994) 400

[5] D.L. Anthony et al., Phys. Rev. Lett. 71 (1993) 759

[6] I.I. Balitskii, V.M. Braun and A.V. Kolesnichenko, Phys. Lett. B242 (1990) 245; B318 (1993) 648

[ 7] J. Ellis and M. Karliner, Phys. Lett. B313 (1993) 131

[ 8] H. Kawamura and T. Uematsu, preprint KUCP-76, hep-ph 9501368

[ 9] V.D. Burkert and B.L. Ioffe, CEBAF preprint, CEBAF-PR-93-034, October 1993

[10] E. Stein, P. Gornicki, L. Mankiewicz, and A. Schaefer, preprint UFTP 380/95, hep-ph 9502323

[11] J. Feltesse, Proceedings of the XXVII International Conference on High Energy Physics, v. I, p. 65, Glasgow, July 20-27, 1994; S.J. Brodsky, SLAC preprint, SLAC-PUB-95-6781

[12] M. Anselmino, S. Ekelin, S. Fredriksson, D.B. Lichtenberg and E. Predazzi, Rev. Mod. Phys. 65 (1993) 1199

[13] T. Schafer, E.V. Shuryak and J.J.M. Verbaarschot, Nucl. Phys. B412 (1994) 143

[14] M. Anselmino, F. Caruso, J.R.T. de Mello, A. Penna Firme and J. Soares, CBPF preprint CBPF-NF-024-94, 1994

[15] M. Anselmino, F. Caruso, E. Leader and J. Soares, Z. Phys. C48 (1990) 689 